\title{
Rainbow meanders and Cartesian billiards
}

\section{Bernold Fiedler}

Institut für Mathematik

Freie Universität Berlin

Arnimallee 3, D-14195 Berlin, Germany

E-mail address: fiedler@math.fu-berlin.de

\section{Pablo Castañeda}

Instituto Nacional de Matemática Pura e Aplicada

Estrada Dona Castorina, 110

Rio de Janeiro, RJ 22460-320, Brasil

- Dedicated to Carlos Rocha on the occasion of his sixtieth birthday, in friendship and gratitude-

\begin{abstract}
In this paper we relate several objects from quite diverse areas of mathematics. Closed meanders are the configurations which arise when one or several disjoint closed Jordan curves in the plane intersect the horizontal axis transversely. The question of their connectivity also arises when evaluating traces in Temperley-Lieb algebras. The variant of open meanders is closely related to the detailed dynamics of Sturm global attractors, i.e. the global attractors of parabolic PDEs in one space dimension; see the groundbreaking work of Fusco and Rocha [FuRo91]. Cartesian billiards have their corners located on the integer Cartesian grid with corner angles of \pm 90 degrees. Billiard paths are at angles of \pm 45 degrees with the boundaries and reflect at half-integer coordinates. We indicate and explore some close connections between these seemingly quite different objects.
\end{abstract}

\section{Introduction}

A simple closed meander is a closed differentiable Jordan curve which is transverse, i.e. nowhere tangent, to the horizontal $x$-axis in the Euclidean plane; see [Ar88, ArVi89] and figure 1.2(a). A closed multi-meander, just called a meander henceforth, consists of finitely many mutually disjoint simple closed meanders. See figure 1.3(a). We assume each Jordan component to intersect the axis at least twice; a Jordan component with exactly 
(a) palaeolithic
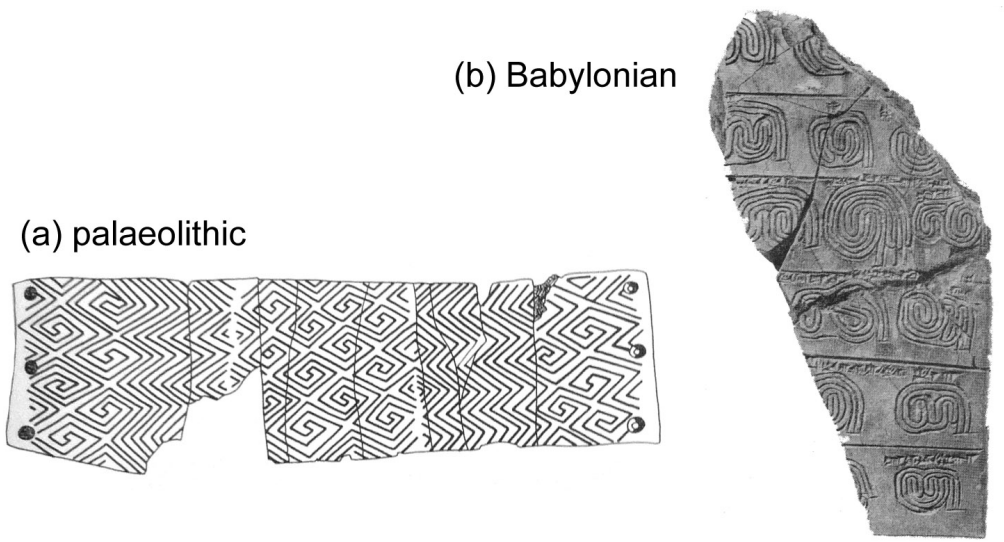

FIGURE 1.1. (a) Meandering patterns on a palaeolithic bracelet dated 15,000 to 18,000 B.C.; see [Gi98], p. 26, Abb. 38. (b) Meandering patterns of entrails of animal sacrifice, Babylonian 1,200 B.C.; see [KB70], p. 214, Abb. 90.

two intersections is called a circle. Open meanders are a variant where the Jordan curves are not assumed to be closed, but of finite and transverse intersection with the $x$-axis.

The intricacies of meander patterns have fascinated mankind, ever since prehistoric times. As examples we mention snake patterns on palaeolithic bracelets, $\sim 15,000$ B.C. [Gi98], Babylonian omina based on meander patterns of entrails, $\sim 1,200$ B.C. [KB70], see figure 1.1, or labyrinthine meanders found in the so-called Nestor Palace, Pylos, 1,200 B.C., and in the floor design of the Cathedral of Chartres, 1,200 A.D. [Sa03], alike.

Merging the two loose ends of a simple open meander into the two loose ends of the $x$-axis, respectively, we have a special case of a closed curve with finitely many transverse self-intersections: the Gauss word problem [Ga1840]; see also section 5.2. More recently, the combinatorics of stamp (now: protein) folding, relations to Termperley-Lieb algebras and statistical physics, and singularity theory have been pursued. See for example the inspiring surveys of Di Francesco et al [dFGG97, dFG05], and section 5.3.

Simple open meanders also arise in descriptions of global attractors of parabolic reaction-advection-diffusion equations of the form

$$
u_{t}=u_{x x}+f\left(x, u, u_{x}\right) \text {, }
$$

say on the unit interval $0<x<1$ with Neumann boundary conditions $u_{x}=0$ at $x=0,1$. Groundbreaking work by Fusco and Rocha [FuRo91] 
(a) simple closed meander

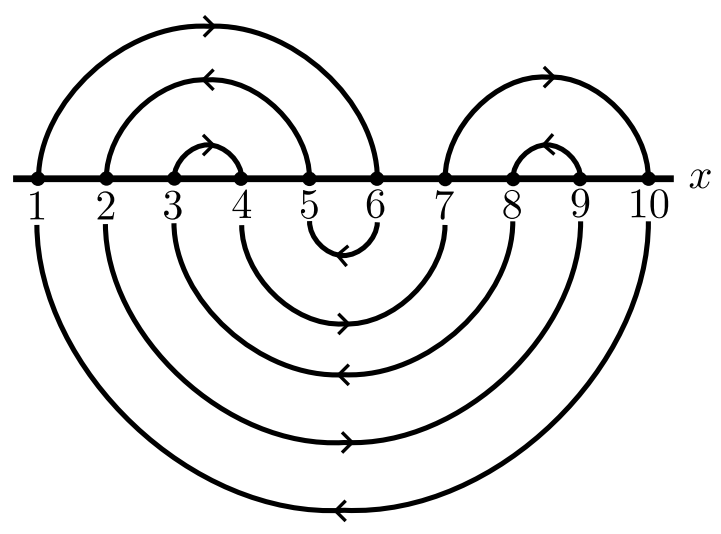

(b) transitive Cartesian billiard

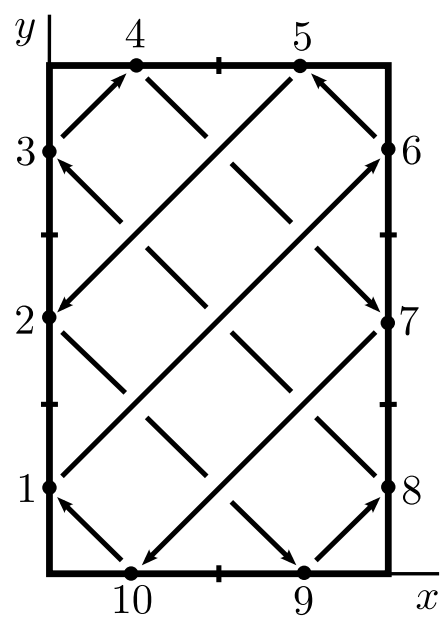

Figure 1.2. (a) A simple, i.e. connected, closed rainbow meander. (b) A corresponding transitive plane Cartesian billiard. The numbered dots in (a) mark intersection points with the $x$-axis and, in (b), corresponding reflection points on the boundary of the associated billiard.

has introduced a permutation characterization of meanders arising from the shooting approach to the equilibrium problem

$$
0=u_{x x}+f\left(x, u, u_{x}\right) .
$$

Indeed, the horizontal $u$-axis in the $\left(u, u_{x}\right)$ phase space of (1.2) corresponds to Neumann boundary conditions, say at $x=1$. See also (5.2), (5.3) below for further detail. The meander curve arises as the image of this axis, starting from "time" $x=0$, when arriving at $x=1$ by the ODE evolution of (1.2). Intersections of the meander with the horizontal uaxis, at $x=1$, indeed correspond to equilibria of (1.1). Transverse, i.e. nontangent, intersections correspond to hyperbolic equilibria. See [ $\mathrm{BrCh} 84$, Ro91, Ro94, Ro07, SmTrWa80] for a detailed account, and [FiSc02, FiRo09] for survey information.

A Cartesian billiard consists of a compact region $B$ in the Euclidean $x y$ plane with polygonal boundary such that each side of the polygon is either horizontal or vertical, and of integer length. See figures 1.2(b), 1.3(b). The corners of the polygon can then be assumed to lie on the standard lattice $\mathbb{Z}^{2}=\mathbb{Z} \times \mathbb{Z}$. As paths in the Cartesian billiard we consider piecewise linear 
(a) closed (multi-)meander

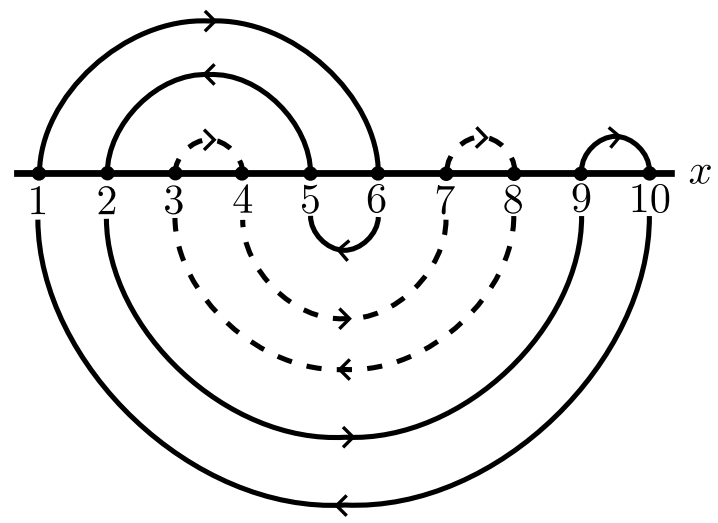

(b) nontransitive Cartesian billiard

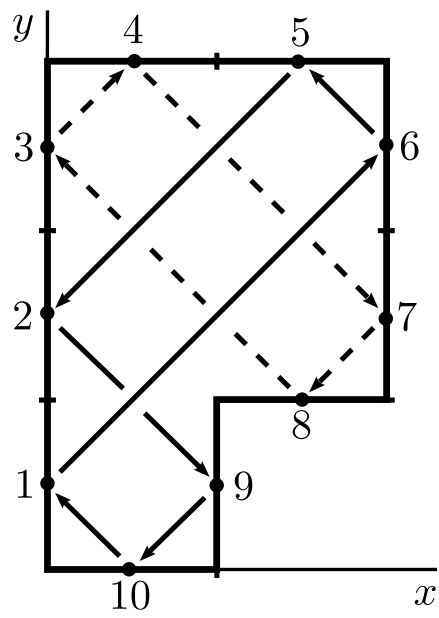

Figure 1.3. (a) A closed rainbow (multi-) meander with two connected components; one distinguished by dashed arches. (b) A corresponding nontransitive plane Cartesian billiard; one flight path distinguished by dashed lines.

flights in $B$ of the form

$$
y \pm x=c \in \mathbb{Z}+\frac{1}{2} .
$$

Subdividing the integer polygon sides into segments of length 1, we thus see how reflection occurs at the half-integer midpoints of the integer boundary segments.

If all flights (1.3) in a Cartesian billiard $B$ define a single closed path, then we call $B$ transitive; see for example figure 1.2(b) above. A rectangle $B$ is transitive if, and only if, its sides $p, q$ are co-prime; see section 6 . For an example of a nontransitive billiard see figure 1.3(b) and, according to section 6 , any $p \times q$ rectangle with sides which are not co-prime.

In the present paper we explore how meanders are related to Cartesian billiards. In particular we investigate several constructions relating meanders to billiards in such a way that simple meanders correspond to transitive billiards.

The remaining part of this introduction is organized as follows. We begin with some background and perspective on meanders in subsection 1.1. Cartesian billiards are formalized, and rotated by $45^{\circ}$, in 1.2 . We formulate our main results in subsection 1.3 and outline the remaining paper in 1.4. 
1.1. Meanders. We collect some terminology and notation concerning meanders, i.e. closed multi-meanders $M$ with simple closed Jordan components $M_{i}$.

Like each component $M_{i}$, the meander $M$ possesses an even number $2 N$ of intersections with the horizontal $x$-axis. Indeed each intersection is transverse, i.e. nontangential, and hence switches sides with respect to the axis. Up to homeomorphism we may assume the intersection points to be at $x=1, \ldots, 2 N$, and the meander curves $M_{i}$ to consist of $2 N$ hemicircle arches with the intersection points as end points: $N$ arches above and $N$ below the axis.

Each of the $N$ arches $a_{k}$ above the axis can also be viewed as a matching pair ( ) of an opening parenthesis "(" and a closing one ")". The usual rules for opening and closing parentheses are equivalent to the mutual nonintersection of the upper arches $a_{k}$. The number of such arch configurations $a_{k}$ above the axis is therefore the $N$-th Catalan number

$$
C_{N}=\frac{1}{N+1}\left(\begin{array}{c}
2 N \\
N
\end{array}\right)
$$

Similarly, the $N$ arches $b_{k}$ below the axis possess $C_{N}$ configurations, and we obtain a total of $C_{2}^{N}$ different meanders, for $2 N$ intersections.

We recall that a circle is a configuration with one upper arch $a$ matching one lower arch $b$ : we obtain a meander component $M_{i}$ which possesses only 2 intersections with the axis. The only meander with $N=1$, for example, consists of a single circle. We call a meander circle-free, if it does not contain any circle component.

Following [dFGG97], we call a meander a rainbow if all lower arches $b_{k}$ are nested. In other words the $k$-th lower arch $b_{k}$ joins the intersection points $k$ and $2 N+1-k$, for $k=1, \ldots, N$. We call a rainbow meander cleaved if none of the upper arches $a_{k}$ joins any intersection point $i \leq N$ to any intersection point $j>N$. Obviously $N$ is even, for cleaved rainbows. Moreover all cleaved rainbows are circle-free.

1.2. Cartesian billiards. For technical convenience in the proofs below we rotate our previous description of a plane Cartesian billiard $B$ by $45^{\circ}$. This makes the billiard flights (1.3) horizontal and vertical, respectively, but produces polygonal boundaries of slopes \pm 1 in the $x y$-plane.

We describe the billiard boundary by the graphs of two continuous functions: the upper boundary $\beta^{+}$and the lower boundary $\beta^{-}$. Let $N \geq 2$. We require $\beta^{ \pm}:[0, N] \rightarrow \mathbb{R}$ to be piecewise linear, for noninteger arguments, 
(a) simple closed meander

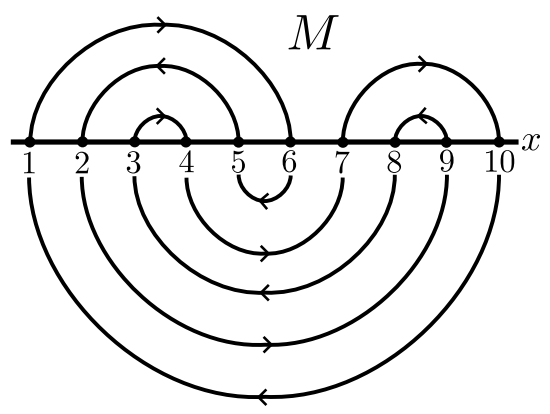

(b) transitive Cartesian billiard

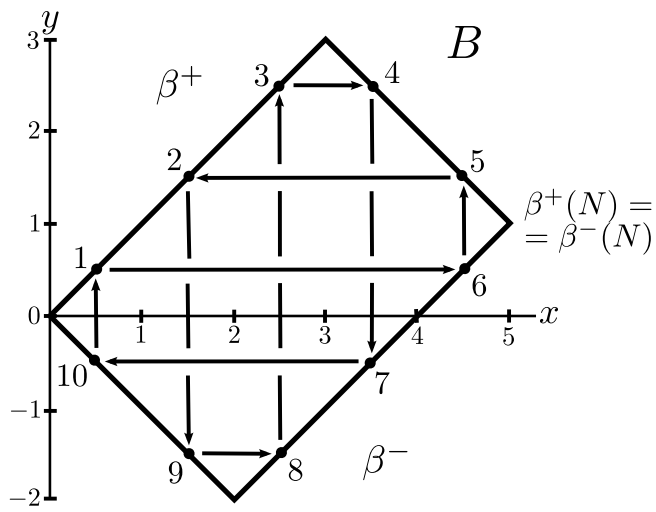

FiguRE 1.4. (a) The simple closed meander $M$ of figure 1.2(a). (b) A transitive billiard $B$ with boundaries $\beta^{ \pm}, N=$ 5 , and associated meander $M=\Phi(B)$. Note how horizontal flights at half-integer constant $y$ in $B$ correspond to the $N=5$ upper arches in $M$, and vertical flights at half-integer constant $x$ correspond to the $N=5$ lower rainbow arches.

and to satisfy for all $0 \leq i<N$

$$
\begin{aligned}
& \beta^{+}(0)=\beta^{-}(0)=0 \text {; } \\
& \beta^{+}(i) \geq \beta^{-}(i) \text {; } \\
& \beta^{+}(i)=\beta^{-}(i) \quad \Longrightarrow \quad \beta^{+}(i \pm 1) \neq \beta^{-}(i \pm 1) ; \\
& \beta^{+}(i+1)=\beta^{+}(i) \pm 1 \quad \text { and } \quad \beta^{-}(i+1)=\beta^{-}(i) \pm 1 \text {; } \\
& \beta^{+}(N)=\beta^{-}(N) \text {. }
\end{aligned}
$$

Of course $\beta^{ \pm}$map integers, and only integers, to integers, by (1.5), (1.8). Note how the linear pieces of $\beta^{ \pm}$each have slopes \pm 1 , by (1.8). Moreover, the upper and lower boundaries may touch, but only at isolated and integer arguments; see (1.6), (1.7). For example $\beta^{ \pm}(1)= \pm 1$ and $\beta^{+}(N-1)=$ $\beta^{-}(N-1)+2$. The billiard region $B$ is the region between the boundaries $\beta^{ \pm}$, i.e.

$$
B=\left\{(x, y) \mid 0 \leq x \leq N, \beta^{-}(x) \leq y \leq \beta^{+}(x)\right\}
$$

To describe the horizontal and vertical billiard flights between reflections at the mid-segments of the boundary we adopt the simplifying notation

$$
n^{\prime}:=n-\frac{1}{2}
$$


(a) closed (multi-)meander

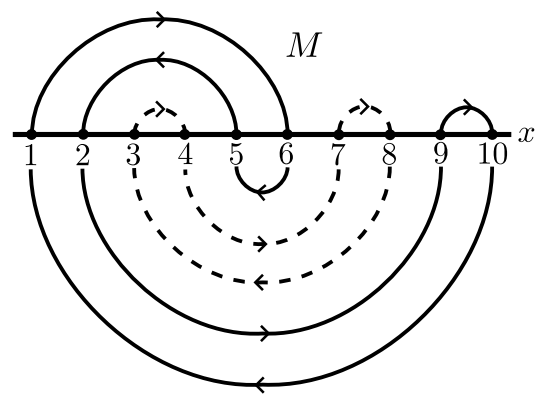

(b) nontransitive Cartesian billiard

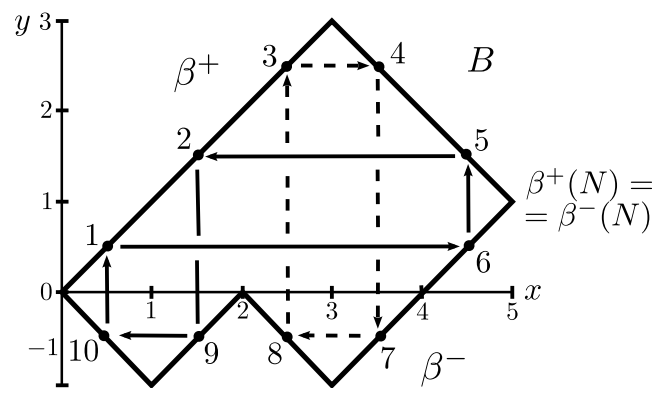

FiguRE 1.5. (a) The simple closed meander $M$ of 1.3(a). (b) A nontransitive billiard $B$ with boundaries $\beta^{ \pm}, N=5$ and associated meander $M=\Phi(B)$. Horizontal/vertical flights at half-integer $y / x$ in $B$ again correspond to the $n=5$ upper/lower arches in $M$, respectively. One flight path/meander component is distinguished by dashing.

for any integer $n$. Then the reflection points of the midsegments are simply the $2 N$ points

$$
\left(i^{\prime}, \beta^{+}\left(i^{\prime}\right)\right) \text { and }\left(i^{\prime}, \beta^{-}\left(i^{\prime}\right)\right)
$$

for $i=1, \ldots, N$. Note that these $2 N$ points are always distinct, because $\beta^{ \pm}$ touch each other only at some isolated and integer arguments. The flights simply preserve the half-integer $x$ - or $y$-value, respectively, and proceed to the next boundary point, where the roles of fixed $x$ or $y$ are reserved.

1.3. Main results. In theorems $1.1-1.3$ below, we formulate our main results on the correspondence between closed meanders $M$ and plane Cartesian billiards $B$. The meanders $M$ possess $2 N$ intersection points with the horizontal $x$-axis, as described in section 1.1. The billiards $B$ are given by continuous boundaries $\beta^{ \pm}:[0, N] \rightarrow \mathbb{R}$, as described in (1.5) - (1.9) of section 1.2 .

Theorem 1.1. Let $N \geq 2$ and consider a plane Cartesian billiard $B$ with boundary functions $\beta^{ \pm}$satisfying properties (1.5) - (1.9) above.

Then $B$ defines a unique associated closed multi-meander

$$
M=\Phi(B)
$$

of $2 N$ intersection points with the horizontal axis. Moreover the associated meander $M$ is a rainbow meander and is circle-free. 
We construct the meander map $\Phi$ in section 2 , explicitly, and prove theorem 1.1 there.

The remaining two main theorems show surjectivity of the map $\Phi$ onto the set of closed circle-free (multi-)meanders with $2 N$ intersection points.

Theorem 1.2. Let $M$ be any closed cleaved rainbow (multi-)meander with $2 N$ intersection points.

Then there exists a plane Cartesian billiard $B$ with boundary $\beta^{ \pm}$such that

$$
\begin{aligned}
& \text { (i) } M=\Phi(B), \quad \text { and } \\
& \text { (ii) } \beta^{ \pm}(N)=0 .
\end{aligned}
$$

Theorem 1.3. Conclusion (i) of the previous theorem holds, more generally, for any closed circle-free rainbow (multi-)meander $M$.

1.4. Outline. We prove theorem 1. $m$ in section $m+1$, for $m=1,2,3$. The proof of theorem 1.1 is by direct interpretation of the billiard paths: the $2 N$ reflection points on the billiard boundary $\beta^{ \pm}$become the intersection points of the meander $M=\Phi(B)$; horizontal flights correspond to the upper arches and vertical flights correspond to lower arches in the bottom rainbow. The proof of theorem 1.2 is by explicit construction of the boundary functions $\beta^{ \pm}$from the opening and closing of upper parentheses in the cleaved rainbow meander. The more intricate proof of theorem 1.3 proceeds by induction, starting from a reduction of circle-free rainbow meanders to cleaved rainbow meanders.

In section 5 we discuss several related constructions on meanders: opening, closing, and conversions to rainbow type which do not affect connectivity. Compactification to meanders on the 2-sphere provides a more embracing view point on some of these constructions and on relations among closed meanders. We also comment briefly on relations to Temperley-Lieb algebras and, in particular, the relation between the Di Francesco trace and rainbow meanders.

We conclude, in section 6 , with an explicit discussion of some elementary rainbow meanders, and a rather innocent-looking open question.

Acknowledgement. The authors gratefully acknowledge generous hospitality at the São Carlos summer conferences of Universidade de São Paulo, and at IMPA, Rio de Janeiro. The author Bernold Fiedler is also much indebted to Paulo Pinto, IST Lisboa, to Holger Reich, FU Berlin, and to Matthias Staudacher, HU Berlin, for helpful discussions on TemperleyLieb algebras. Bruno Leukert has provided expert background on Babylonian clay tablets and their omina. Skillful typesetting was cheerfully carried through numerous versions by Ulrike Geiger. Most of all, however, 
Bernold Fiedler is indebted to Carlos Rocha for deep and lasting friendship of many years.

This work was partially supported by the Deutsche Forschungsgemeinschaft via SFB 647 "Space - Time - Matter", and by FAPERJ via the fellowship grant E-26/102.474/2010.

\section{Proof of theorem 1.1: from billiards to rainbow meanders}

In this section we prove theorem 1.1: given a plane Cartesian billiard $B$ we construct a meander $M$ and thus define the meander map $M=\Phi(B)$ of (1.13).

We recall that the billiard $B$ is given by its continuous boundary functions $\beta^{ \pm}:[0, N] \rightarrow \mathbb{R}$, which satisfy the boundary properties (1.5) - (1.9). For $i=1, \ldots, N$ in (1.12) we label the $2 N$ half-integer reflection points

$$
\begin{aligned}
\left(i^{\prime}, \beta^{+}\left(i^{\prime}\right)\right) & \text { by } \quad m=i \in\{1, \ldots, N\}, \quad \text { and } \\
\left(i^{\prime}, \beta^{-}\left(i^{\prime}\right)\right) & \text { by } \quad n=2 N+1-i \in\{N+1, \ldots, 2 N\},
\end{aligned}
$$

clockwise along the billiard boundary.

To construct a meander from the vertical and horizontal billiard flights through these reflection points, we consider vertical flights first. Let each vertical flight $x=$ const. $=i^{\prime} \in \mathbb{Z}+\frac{1}{2}$ between $\left(i^{\prime}, \beta^{+}\left(i^{\prime}\right)\right)$ and $\left(i^{\prime}, \beta^{-}\left(i^{\prime}\right)\right)$ define a lower arch between the labels $m=i$ and $n=2 N+1-i$. Obviously this defines $N$ nested lower arches, as required in a rainbow.

To define the upper arches we consider a nonempty connected horizontal flight interval $y=$ const. $\in \mathbb{Z}+\frac{1}{2}$ within the compact billiard region $B$. Note

how the reflecting endpoints $\left(i_{1}^{\prime}, \beta^{\iota_{1}}\left(i_{1}^{\prime}\right)\right)$ and $\left(i_{2}^{\prime}, \beta^{\iota_{2}}\left(i_{2}^{\prime}\right)\right)$ may belong to the same boundary graph, $\iota_{1}=\iota_{2} \in\{+,-\}$, or to opposite boundary graphs, $\iota_{1} \neq \iota_{2}$. Nevertheless the horizontal flight defines an upper hemicircle arch between the appropriate labels (2.1) of its reflecting endpoints.

It is already obvious that the resulting arch diagram is circle-free. Indeed proceed indirectly and consider the two successive reflection points in the billiard $B$ which correspond to the intersection points of a hypothetical circle with the $x$-axis. Then the two reflection points coincide in $B$ : in their $x$-coordinates by the vertical flight of the lower hemicircle, and in their $y$-coordinates by the horizontal flight of the upper hemicircle. By costruction, however, two successive reflection points in $B$ cannot coincide.

Noting how upper and lower arches alternate, as do horizontal and vertical flights in the billiard $B$, it only remains to show that the resulting upper arches are disjoint. Then the constructed arch configuration $M=\Phi(B)$ is a (multi-)meander, indeed, and the proof of theorem 1.1 will be complete. 
We show that two distinct upper arches $a$, from $A_{1}$ to $A_{2}>A_{1}$, and $\hat{a}$, from $\hat{A}_{1}$ to $\hat{A}_{2}$, do not intersect. Without loss of generality we may assume that the meander intersection $\hat{A}_{1}$ is between $A_{1}$ and $A_{2}$ on the $x$-axis. We have to show that $\hat{A}_{2}$ is between $A_{1}$ and $A_{2}$, likewise. We identify the four intersections with their corresponding reflection points on the clockwise boundary curve $\beta=\beta^{+} \cup \beta^{-}$of the billiard $B$. We open up $\beta$, and $B$, slightly at touching point integers $\left(i, \beta^{ \pm}(i)\right), 0<i<N$ without obstructing any part of the billiard path. Let $\tilde{B}$ denote this slightly modified billiard, now with closed Jordan curve boundary $\tilde{\beta}$. The unchanged horizontal flights $A_{1} A_{2}$ and $\hat{A}_{1} \hat{A}_{2}$ in $\tilde{B}$ are disjoint, by construction: either their half-integer $y$-values disagree, or else the flights belong to different $\tilde{B}$-components of the same half-integer $y$-level. Consider the closed Jordan curve which consists of the horizontal $A_{1} A_{2}$ flight and the piece of the boundary circle $\tilde{\beta}$ oriented clockwise from $A_{1}$ to $A_{2}$. Since $\hat{A}_{1}$ lies on that boundary piece, so does $\hat{A}_{2}$, by the Jordan curve theorem in $\tilde{B}$ and because the horizontal flights $A_{1} A_{2}$ and $\hat{A}_{1} \hat{A}_{2}$ do not intersect. This proves that $M=\Phi(B)$ is a meander, and completes the proof of theorem 1.1.

\section{Proof of theorem 1.2: from cleaved rainbow meanders to billiards}

In this section we prove theorem 1.2: given a cleaved rainbow meander $M$ with $2 N$ axis intersections, we explicitly construct a plane Cartesian billiard $B$ via its defining continuous boundary functions $\beta^{ \pm}:[0, N] \rightarrow \mathbb{R}$. We construct $\beta^{ \pm}$with properties $(1.5)-(1.9)$ and such that $\beta^{ \pm}(N)=0$. In terms of the meander map $\Phi$ of theorem 1.1, as constructed in the previous section, we then show $M=\Phi(B)$ as required in theorem 1.2.

We define the upper billiard boundary $\beta^{+}$first. Let $\beta^{+}(0)=0$, as required by (1.5). We satisfy (1.7) by the recursive definition

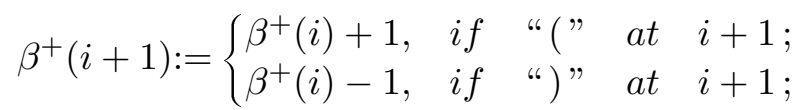

for $i=0, \ldots, N-1$. Here we have represented the upper arches of the rainbow meander $M$ to the left of the cleavage by parenthesis expressions "(", ")" at each intersection point $i+1=1, \ldots, N$, as in the Catalan counting (1.4).

We use the upper arches to the right of the meander cleavage, right to left, to define the lower boundary

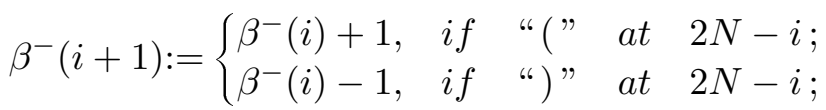


again for $i=0, \ldots, N-1$ and starting at $\beta^{-}(0):=0$. We complete the definition of $\beta^{ \pm}$by linear interpolation.

We check properties (1.5) - (1.9) of $\beta^{ \pm}$. Obviously properties (1.5), (1.8) hold by construction. Because $M$ is cleaved at $N, N+1$, all opened parentheses at $i=1, \ldots, N-1$ have been closed when we reach $i=N$. Therefore (3.1) implies $\beta^{+}(N)=0$. Reading right to left, on the right of the cleavage, analogously proves $\beta^{-}(N)=0$ by (3.2). This proves property (1.9) and claim (ii) of theorem 1.2. Since only open parentheses can close we observe

$$
\beta^{+}(i) \geq 0 \geq \beta^{-}(i)
$$

for all $i=0, \ldots, N$. This proves property (1.6). Moreover (1.8) and (3.3) imply $\beta^{+}>0$ and $\beta^{-}<0$ at integer neighbors of zeros of $\beta^{ \pm}$. This proves property (1.7) and shows how the boundaries (3.1), (3.2) indeed define a plane Cartesian billiard $B$.

It remains to show $\Phi(B)=M$ for the meander map $\Phi$ constructed in the previous section. Since $\Phi(B)$ is always a rainbow, by construction, we only have to address the upper part. Flipping the billiard $B$ upside down, by reflection through the $x$-axis, the roles of $\beta^{ \pm}$interchange and the meander $\Phi(B)$ is reflected through the vertical axis $x=N+\frac{1}{2}$. Therefore it is sufficient to consider the upper arches to the left of the cleavage of $M$, and the horizontal flights $0<y \in \mathbb{Z}+\frac{1}{2}$ of the billiard $B$ which only involve the boundary $\beta^{+}$.

Let $j_{1} j_{2}$ denote any upper arch of $M$ to the left of the cleavage, $1 \leq j_{1}<$ $j_{2} \leq N$. The corresponding parenthesis pair "( " at $j_{1}$ and ")" at $j_{2}$ then implies slope +1 of $\beta^{+}$at $j_{1}^{\prime}=j_{1}-\frac{1}{2}$ and slope -1 at $j_{2}^{\prime}$, by construction (3.1). The fact that ")" at $j_{2}$ is the closing match of the parenthesis "(" opened at $j_{1}$ implies $\beta^{+}\left(j^{\prime}\right)>\beta^{+}\left(j_{1}^{\prime}\right)=\beta^{+}\left(j_{2}^{\prime}\right)$ for all $j_{1}<j<j_{2}$, again by (3.1). In other words, the unobstructed horizontal billiard flight between the boundary reflection points $\left(j_{1}^{\prime}, \beta^{+}\left(j_{1}^{\prime}\right)\right)$ and $\left(j_{2}^{\prime}, \beta^{+}\left(j_{2}^{\prime}\right)\right)$ in $B$ at halfinteger level $y=\beta^{+}\left(j_{1}^{\prime}\right)=\beta^{+}\left(j_{2}^{\prime}\right)$ corresponds precisely to the upper arch between the intersection points $j_{1}$ and $j_{2}$ in the meander $M$. This bijection between the $N / 2$ horizontal flights in $B$ at positive half-integer levels of $\beta^{+}$ and the upper arches of the meander $M$ to the left of the cleavage proves $M=\Phi(B)$ and completes the proof of theorem 1.2.

\section{Proof of theorem 1.3: from circle-free rainbow meanders to billiards}

In this section we prove theorem 1.3: given any circle-free rainbow (multi) meander $M$ with $2 N$ axis intersections, we recursively construct a plane Cartesian billiard $B$ such that $M=\Phi(B)$. 
We proceed by reduction to a cleaved rainbow. Starting from the circlefree non-cleaved rainbow $M=M_{0}$ we construct rainbows $M_{1}, M_{2}, \ldots$ by successively removing the topmost upper $\operatorname{arch} j_{1} j_{2}$ across the cleavage. In other words

$$
j_{1} \leq N<j_{2}
$$

and $j_{1}$ is minimal (and hence $j_{2}$ maximal) among all arches $j_{1} j_{2}$ with this property. Of course we also remove one arch from the lower rainbow, in parallel. At each reduction step from $M_{m}$ to $M_{m+1}$ we show that $M_{m+1}$ is circle-free, if $M_{m}$ was circle-free; see lemma 4.1 below. Moreover we show that $M_{m}=\Phi\left(B_{m}\right)$ arises as the meander of a billiard, if $M_{m+1}=\Phi\left(B_{m+1}\right)$ does; see lemma 4.2 below. Since $M_{0}$ is circle-free, the descent $M_{0}, M_{1}, \ldots$ by lemma 4.1 cannot terminate at the empty meander but must terminate at a cleaved rainbow meander $M_{n}$, as soon as all the $n$ arches across the "cleavage" $N, N+1$ in $M_{0}$ have been removed. Since $M_{n}$ is a cleaved rainbow, theorem 1.2 and the explicit construction in section 3 provide a plane Cartesian billiard $B_{n}$ such that $M_{n}=\Phi\left(B_{n}\right)$ is the associated cleaved rainbow meander. By lemma 4.2 below we may then ascend back to $M=M_{0}=\Phi\left(B_{0}\right)$ by induction, and theorem 1.3 will be proved.

We only need to formulate lemmas 4.1 and 4.2 for a single reduction step, say from $M_{0}$ to $M_{1}$. To fix notation we label the intersection points of the reduced meander $M_{1}$ with the horizontal axis by $1, \ldots, 2 N$, as usual. For the extended meander $M_{0}$ we append labels 0 and $2 N+1$, symmetrically, and denote the added upper arch by $j_{1} j_{2}$ as in (4.1), with $0 \leq j_{1} \leq N<$ $j_{2} \leq 2 N+1$.

Lemma 4.1. If the extended rainbow meander $M_{0}$ is circle-free, then so is the reduced rainbow meander $M_{1}$.

\section{Proof.}

We prove the contrapositive. Suppose the reduced rainbow $M_{1}$ possesses a circle, i.e. an upper arch $k_{1} k_{2}$ with $k_{2}=2 N+1-k_{1}$. We claim the extended rainbow $M_{0}$ possesses the same circle.

Indeed we may fix notation such that $k_{1} \leq N<k_{2}$. Minimality (4.1) of $j_{1}$ in the added upper arch $j_{1} j_{2}$ of $M_{0}$ implies $j_{1}<k_{1}$ and $j_{2}>k_{2}$. In particular the upper arch $k_{1} k_{2}$ of the reduced rainbow $M_{1}$ appears as an upper arch in the extended rainbow $M_{2}$ with the same labels. Therefore the extended rainbow $M_{0}$ possesses the same circle $k_{1} k_{2}$ and the lemma is proved.

The converse of lemma 4.1 fails, of course: we may always add a circle by a topmost upper arch $j_{1}=0, j_{2}=2 N+1$, in the extended meander $M_{0}$. 


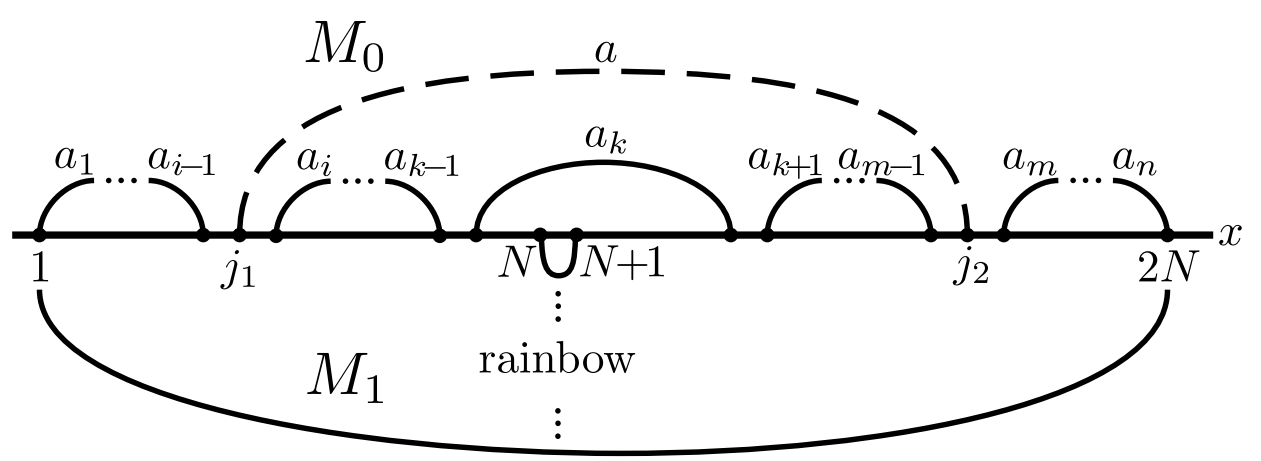

FiguRE 4.1. Extended rainbow meander $M_{0}$ and reduced rainbow meander $M_{1}$. The dashed topmost upper arch $a=$ $j_{1} j_{2}$ of $M_{0}$ overarching the cleavage of the lower rainbow at $N, N+1$ is removed in $M_{1}$.

Lemma 4.2. Let the extended rainbow meander $M_{0}$ be circle-free and assume that the circle-free reduced rainbow meander $M_{1}$ possesses a billiard representation $M_{1}=\Phi\left(B_{1}\right)$ by a plane Cartesian billiard $B_{1}$.

Then the extended rainbow meander $M_{0}$ also possesses a billiard representation $M_{0}=\Phi\left(B_{0}\right)$ by a plane Cartesian billiard $B_{0}$.

\section{Proof.}

We divide the proof into four steps. In a first step, we fix some notation for the upper arches of the rainbow meanders $M_{0}, M_{1}$, and for the given billiard $B_{1}$ representing the reduced rainbow meander $M_{1}$. In step 2 we identify the topmost upper arches of $M_{1}$ by their corresponding horizontal flights in $B_{1}$. We also focus on the most typical subcase of the actual proof. Step 3 contains the crucial construction to insert a new topmost upper arch $a=j_{1} j_{2}$, across the rainbow cleavage at $N, N+1$, for the extended rainbow $M_{0}$ by inserting a new horizontal flight in $B_{1}$. This will construct the billiard $B_{0}$. In step 4 we check that $B_{0}$ is indeed a plane Cartesian billiard with $\Phi\left(B_{0}\right)=M_{0}$, as claimed.

\section{Step 1: Notation.}

In figure 4.1 we have sketched the sequence of topmost upper arches of the reduced rainbow meander $M_{1}$ and the extended rainbow meander $M_{0}$. The topmost upper arches of $M_{1}$ are labeled $a_{1}, \ldots, a_{n}$ from left to right; they correspond to outermost pairs of matching parentheses " (...)". The dashed additional arch $a=j_{1} j_{2}$ of $M_{0}$ overarches $a_{i}, \ldots, a_{m-1}$ 
(a) reduced billiard $B_{1}$

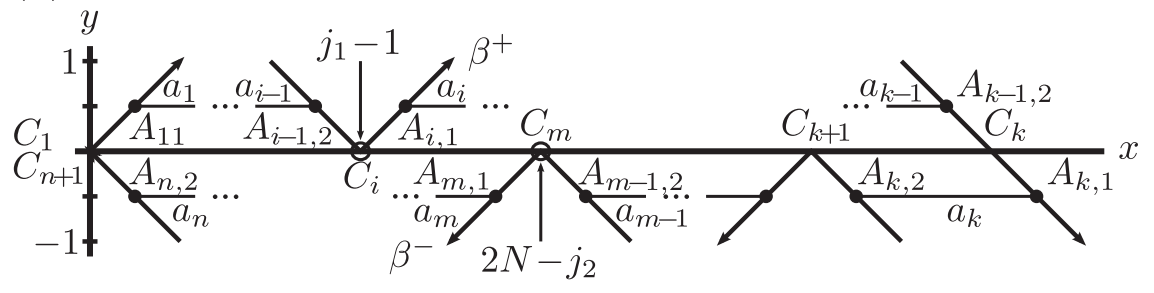

(b) extended billiard $B_{0}$

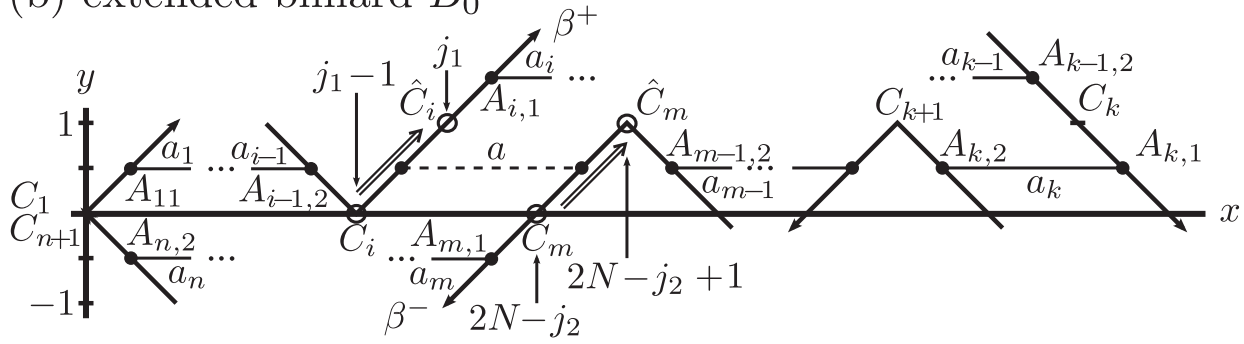

FiguRE 4.2. (a) Billiard representation $\Phi\left(B_{1}\right)=M_{1}$ of the reduced rainbow meander $M_{1}$ of figure 4.1. (b) Billiard representation $\Phi\left(B_{0}\right)=M_{0}$ for the extended rainbow meander $M_{0}$ of figure 4.1. Note the shift by $(1,1)$ of the billiard boundary $B_{1}$, from $C_{i}$ to $C_{m}$, to construct the part from $\hat{C}_{i}$ to $\hat{C}_{m}$ of the extended billiard boundary $B_{0}$ for the extended meander $M$.

with $1 \leq i \leq m \leq n$, so that the topmost upper arches of $M_{0}$ are $a_{1}, \ldots, a_{i-1}, a, a_{m}, \ldots, a_{n}$. The additional arch $a$ is the unique topmost upper arch of the extended rainbow meander $M_{0}$ which overarches the lower rainbow cleavage at positions $N, N+1$.

In figure 4.2(a) we sketch one possible configuration of the boundaries $\beta^{ \pm}$of the billiard $B_{1}$ which represents the reduced rainbow meander $M_{1}=$ $\Phi\left(B_{1}\right)$, by assumption. We discuss the remaining cases in step 2. Specifically we have assumed $C_{k}>0$ to be the smallest positive integer such that $\beta^{+}\left(C_{k}\right)=0$ and $\beta^{+}\left(C_{k}+1\right)=-1$. We have also assumed $\beta^{-}(x) \leq 0$ for $0 \leq x \leq C_{k}+1$. The interval components of the horizontal flights at $y=\frac{1}{2}$ in $B_{1}$ are labeled $a_{1}, \ldots, a_{k-1}$ from left to right. The analogous flights at $y=-\frac{1}{2}$ are labeled $a_{k}, \ldots, a_{n}$, from right to left. In step 2 we will see how $a_{\ell}$ labels corresponding topmost upper arches and horizontal flights in $M_{1}$ and $B_{1}$. Again we join the boundaries $\beta=\beta^{+} \cup \beta^{-}$in clockwise direction; so that the path $\beta$ starts from the origin in direction $\beta^{+}$and returns to the 
origin after having traversed $\beta^{-}$. In this order, we denote the end points of flight $a_{\ell}$ by $A_{\ell, 1}<A_{\ell, 2}$. Along the boundary path $\beta=\beta^{+} \cup \beta^{-}$we have also labeled zeros by $C_{1}=0<C_{2}<\cdots<C_{k}$, left to right, along the path $\beta^{+}$, and $C_{k+1}>\cdots>C_{n+1}=0$, right to left, along the return path $\beta^{-}$. The zeros at $C_{i}$ and $C_{m}$ of $\beta^{ \pm}$occur at $x$-values $j_{1}-1$ and $2 N-j_{2}$. They indicate where we plan to insert the additional horizontal flight of the billiard $B_{0}$ corresponding to the additional arch $a=j_{1} j_{2}$ of the extended meander $M_{0}$, in step 3.

$\underline{\text { Step 2: Topmost arches and dropped cases. }}$

Let us first assume that the plane Cartesian billiard $B_{1}$ with reduced rainbow meander $M_{1}=\Phi\left(B_{1}\right)$ satisfies

$$
\beta^{+} \geq 0 \geq \beta^{-},
$$

and hence in particular $\beta^{+}(N)=\beta^{-}(N)=0$. Then horizontal flights at any half-integer levels $y \in \mathbb{Z}+\frac{1}{2}$ begin and terminate on $\beta^{ \pm}$for $\pm y>0$, but never run between $\beta^{+}$and $\beta^{-}$. Therefore (4.2) implies that the reduced rainbow meander $M_{1}$ is cleaved. Moreover, the flights with $y=+\frac{1}{2}$ provide the topmost upper arches to the left of the cleavage at $N, N+1$, and $y=-\frac{1}{2}$ provides the topmost upper arches to the right. Indeed the upper arches $a_{1}, a_{2}, \ldots$ start at the meander intersection $A_{11}=1$ with the horizontal axis, which corresponds to the billiard point $\left(1^{\prime}, \beta^{+}\left(1^{\prime}\right)\right)$ with $1^{\prime}=\frac{1}{2}$ and $y=\frac{1}{2}$. Along $\beta^{+}$we successively run into subsequent flights at $y=\frac{1}{2}$, from $a_{i-1}$ to $a_{i}$, because $\beta^{+}=0$ at $C_{i}$. Along $\beta^{-}$, a similar argument applies, down from $a_{n}, a_{n-1}, \ldots$ at level $y=-\frac{1}{2}$.

Let us next consider the case that $\beta^{+}$or $\beta^{-}$do attain values where $\beta^{+}<0$ or $\beta^{-}>0$. In figure $4.2(\mathrm{a})$ we have considered the case that $\beta^{+}<0$ occurs first, along the $x$-axis. In the opposite case $\beta^{-}>0$ the boundary at $C_{k}$ simply possesses positive slope and belongs to $\beta^{-}$, already. In the following we will suppress this analogous case, along with the cleaved meander case (4.2) where $C_{k}=N$ and $A_{k-1,2}$ belongs to $\beta^{+}$but $A_{k, 1}$ belongs to $\beta^{-}$. We thus assume

$$
\begin{array}{ll}
\beta^{+}\left(C_{k}+1\right)=-1, & \text { and } \\
\beta^{+}(x) \geq 0 \geq \beta^{-}(x) & \text { for } \quad 0 \leq x \leq C_{k} .
\end{array}
$$

To insert the dashed arch $a=j_{1} j_{2}$, when extending the meander $M_{1}$ to $M_{0}$, we have to insert an additional half-integer reflection point near the zeros $C_{i}$ of $\beta^{+}$, for $j_{1}$, and $C_{m}$ of $\beta^{-}$for $j_{2}$. 
We first observe that $C_{i} \neq C_{m}$. Indeed $C_{i}=C_{m}$ would imply that the labels $A_{\ell, \kappa}$ satisfy

$$
A_{i, 1}=2 N+1-A_{m-1,2},
$$

and are hence positioned symmetrically to the cleavage of the lower rainbow at $N, N+1$. Therefore the arch $a$ of $M_{0}$ would belong to a circle, but $M_{0}$ is assumed circle-free. This proves $C_{i} \neq C_{m}$.

It remains to consider the cases $C_{i}>C_{m}$ and $C_{i}<C_{m}$. By reflection of the billiards $B_{0}, B_{1}$ through the horizontal axis, and of the meanders $M_{0}$, $M_{1}$ through the vertical axis at $x=N+\frac{1}{2}$, however, we may focus on the case

$$
C_{i}<C_{m}
$$

of figure 4.2 for the remaining proof.

Step 3: Extending the billiard from $B_{1}$ to $B_{0}$.

In the previous step we have reduced the proof to the reduced meander case $M_{1}=\Phi\left(B_{1}\right)$ of figure 4.1 with the reduced billiard of figure $4.2(\mathrm{a})$. We now keep the billiard boundary $\beta^{+}$fixed, from $C_{1}=0$ to $C_{i}$, and we fix $\beta^{-}$ from $C_{m}$ to $C_{n+1}=0$. The remaining boundary part of $\beta=\beta^{+} \cup \beta^{-}$from $C_{i}$ to $C_{m}$ we move by the vector $(1,1)$, i.e. one step to the right and one step up. We interpolate the resulting gaps at $C_{i}$ and $C_{m}$ linearly, i.e. with slope +1 . See figure $4.2(\mathrm{~b})$ for the resulting extension $B_{0}$ of the billiard $B_{1}$.

$$
\text { Step 4: } \Phi\left(B_{0}\right)=M_{0} .
$$

It is straightforward to check properties $(1.5)$ - (1.9) for the extension of $\beta^{ \pm}$constructed above, either geometrically or algebraically, to see that $B_{0}$ is indeed a plane Cartesian billiard. Indeed, the partial shift by $(1,1)$ preserves the closing properties (1.5), (1.9), the slope property (1.8), the ordering (1.6), and does not introduce any new tangencies (1.7).

The horizontal flights in $B_{1}$ are all preserved by the extension to $B_{0}$, although the half-integer $y$-levels increase by 1 in the shifted part. Check the neighboring reflection points $A_{i-1,2}$ and $A_{i, 1}$ of the newly inserted reflection point $j_{1}$ at $\left(C_{i}, 0\right)+\frac{1}{2} \cdot(1,1)$, and likewise of the neighbors $A_{m-1,2}$, $A_{m, 1}$ of $j_{2}$ at $\left(C_{m}, 0\right)+\frac{1}{2} \cdot(1,1)$, along the boundary $\beta$. We see how the extended meander $\Phi\left(B_{0}\right)$ only differs from $M_{1}$ by a newly inserted upper arch $a=j_{1} j_{2}$ overarching $a_{i}, \ldots, a_{m-1}$ from the end $A_{i-1,2}$ of $a_{i-1}$ to the beginning $A_{m, 1}$ of $a_{m}$. Therefore $\Phi\left(B_{0}\right)=M_{0}$.

Up to the careful consideration of a few remaining special cases like $m=k$ or $i=k$, or the cleavage case $C_{k}=N$ and the analogous case 
$C_{k} \in \beta^{-}$, as discussed in step 2 , this completes the proof of the lemma, and of theorem 1.3.

\section{Morphing meanders}

In the previous sections we have investigated the correspondence between circle-free rainbow meanders and their associated plane Cartesian billiards. In the four subsections below we widen the scope of our results to include more general meander configurations. In subsection 5.1 we discuss the relation between open and closed meanders, with an eye on the open connected, i.e. simple, meanders which arise in parabolic PDEs of Sturm type; see (1.2). In 5.2 we study closed meanders on the standard 2-sphere $S^{2}$, the one-point compactification of the Euclidean meander plane, to indicate the resulting equivalences of closed meanders. The role of closed meanders in Temperley-Lieb algebras is briefly sketched in 5.3, relating the connectivity of rainbow meanders with Di Francesco's notion of a trace in TemperleyLieb algebras. In subsection 5.4 we conclude with two constructions of rainbow meanders, from general closed (multi-)meanders. Both constructions preserve connectivity. This completes our objective of relating the connected open meanders, as they arise in Sturmian parabolic PDEs, with connectivity of circle-free rainbow-meanders, traces in Temperley-Lieb algebras, and transitivity of plane Cartesian billiards.

5.1. Opening and closing meanders and billiards. We begin with a PDE example from the class of global attractors of the scalar parabolic equations

$$
u_{t}=u_{x x}+f\left(x, u, u_{x}\right)
$$

on the unit interval $0<x<1$ under Neumann boundary conditions $u_{x}=0$ at $x=0$ and $x=1$. Based on [FiRo96] the global attractor sketched in figure 5.1(a) has been identified as one of sixteen genuinely different examples with 9 hyperbolic equilibria in this class, see [Fi94]. The particular example does not arise for $f=f(u)$; see [FiRoWo11].

In figure 5.1(a) we sketch the 9 hyperbolic equilibria $v=v(x)$ of $(5.1)$, i.e. the solutions of the second order ODE

$$
0=v_{x x}+f\left(x, v, v_{x}\right)
$$

again with Neumann boundary conditions $v_{x}=0$ at $x=0,1$. Each equilibrium $v$ is indicated by a dot, labeled $0, \ldots, 8$. The Morse indices $i$, alias the unstable dimensions, alias the number of strictly positive Sturm-Liouville eigenvalues of the linearization of (5.1) at $v$, are given by $i=0$ for labels 0,8 (stability); $i=1$ for labels $1,5,7$; and $i=2$ for labels $2,4,6$. Label 3 indicates the only 3 -dimensionally unstable equilibrium, central to the 
(a) Sturm global attractor

(b) open meander

(c) closed meander
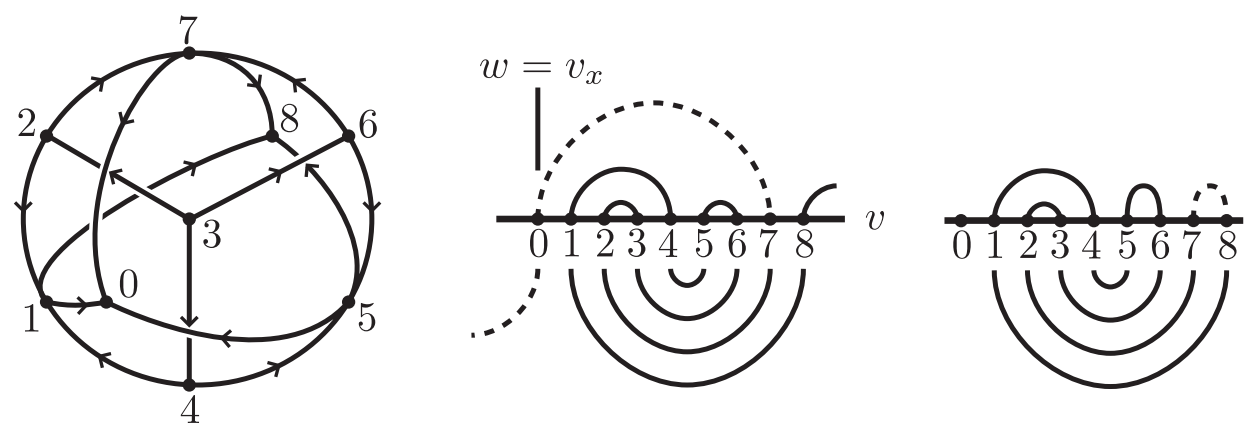

Figure 5.1. (a) Sturm global attractor of PDE (5.1) with 9 hyperbolic equilibria $v$, labeled by $0, \ldots, 8=2 N$. Onedimensional heteroclinic orbits between equilibria of adjacent Morse index are indicated by arrows. (b) Open meander, at $x=1$, which leads to the 9 equilibrium solutions $v$ by shooting from the Neumann boundary condition at $x=0$, see (5.2), (5.3). (c) Closed meander which arises by replacing the dashed upper arch $(0,7)$, in (b), with the new dashed upper $\operatorname{arch}(7,8)$.

global attractor which is a closed 3-ball. For more details on the (Schoenflies) sphere geometry in global attractors of (5.1) see [FiRo13]. In figure 5.1 (a) we sketch the full attractor geometry by heteroclinic orbit solutions $u(t, x) \rightarrow v_{ \pm}(x)$ of (5.1), which tend to different equilibria $v_{ \pm}$of adjacent Morse indices, for $t \rightarrow \pm \infty$.

In figure 5.1(b) we sketch the shooting curve associated to the Neumann boundary value problem (5.2). More precisely we rewrite (5.2) as a nonautonomous first order system

$$
\begin{aligned}
v_{x} & =w \\
w_{x} & =-f(x, v, w)
\end{aligned}
$$

with Neumann boundary values $w=0$ at $x=0,1$. Solving (5.3) with the $v$-axis $\{w=0\}$ as a set of initial conditions, at $x=0$, we obtain an open meander curve as in figure $5.1(\mathrm{~b})$, at $x=1$. Transverse intersections of the open meander with the $v$-axis $\{w=0\}$ at $x=1$ indicate hyperbolic equilibria $u(t, x) \equiv v(x)$ of (5.3). Obviously, the open meander will be simple, i.e. connected, if we assume global solvability of (5.3) for initial conditions $w=0$ at $x=0$, and all $0 \leq x \leq 1$. 
In figure 5.1(c) we sketch how to relate the open meander of figure 5.1 (b) to a closed meander. More generally consider any plane open multimeander $M$ with exactly one unbounded component. Leaving the PDE notation of $(5.1)-(5.3)$ behind we return to calling the horizontal axis $x$, again, and label the $2 N+1$ transverse intersections by $0, \ldots, 2 N$. We also assume the unbounded component of the meander $M$ to cross from the lower to the upper half plane at labels 0 and $2 N$, as indicated in figure $5.1(\mathrm{~b})$. We can then replace the topmost upper arch $(0, m)$ of $M$ by a new topmost upper arch $(m, 2 N)$ to close the meander. We omit the intersection point 0 in the resulting closed meander $\tilde{M}$. Alternatively we may also omit $2 N$ and replace the lowest bottom arch $(n, 2 N)$ by a new lowest bottom arch $(0, n)$, instead. Either way, $M$ gives rise to a total of two associated closed meanders $\tilde{M}$ with $2 N$ intersections and with the same number of connected components as $M$. In particular simple meanders stay simple under closing.

Conversely we may think of opening closed meanders $\tilde{M}$ on $1, \ldots, 2 N$. Suppose we aim for an open meander $M$ with upward crossings of the unbounded ends at $0,2 N$ such that $\tilde{M}$ arises from $M$, in turn, as above. Then we just have to replace the topmost upper arch $(m, 2 N)$ of $\tilde{M}$ by $(0, m)$. Similarly we can revert the closing of the lowest bottom arch $(1, n)$ of $M$ to become an opening. In this way we can view opening and closing as inverse constructions which preserve the number of connected components. For a more general view point see section 5.2 on $S^{2}$ compactified meanders.

Of course we may consider other variants of opening and closing, as well. If the unbounded ends cross downwards at $0,2 N$, instead, we may flip the above considerations through the $x$-axis. If the unbounded ends are in the same half-plane we may just join them to form a new arch in that half-plane, for closing. Uniqueness of inverse openings will be lost, in general. If the unbounded ends are in opposite half-planes, but not crossing at $0,2 N$, it may not be possible to close the meander $0, \ldots, 2 N$ with just $2 N$ crossings. Adding another crossing -1 or $2 N+1$, however, a closing with $2 N+2$ crossings is always possible.

Opening and closing plane Cartesian billiards is equally straightforward; see figure 5.2. To accommodate unbounded meander ends, which enter the upper half plane at $0,2 N$, we attach a $1 \times \frac{1}{2}$ rectangle $R$ at the original polygon edge interval of length 1 bisected by $2 N$. (We call the rectangle $1 \times \frac{1}{2}$ even though the (Euclidean) lengths of its sides require a suppressed factor of $\sqrt{2}$.) We relabel the new corners as 0 and $2 N$, respectively. Since these are the only corners at half-integer levels of $x, y$, they mark the start and end points of a billiard path which corresponds to the unbounded component of the open meander; see figures 5.1(b) and 5.2(b). 


\section{(a) closed billiard}

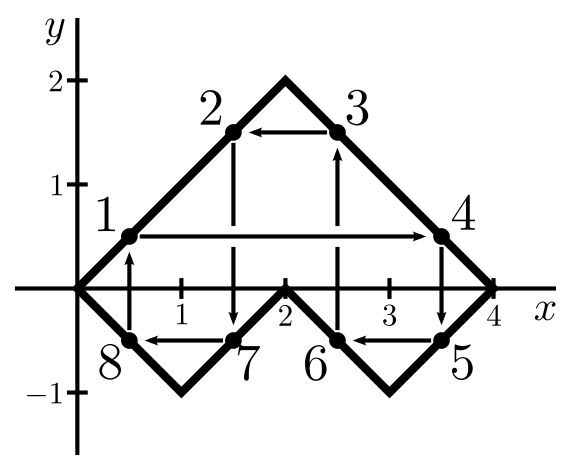

(b) open billiard

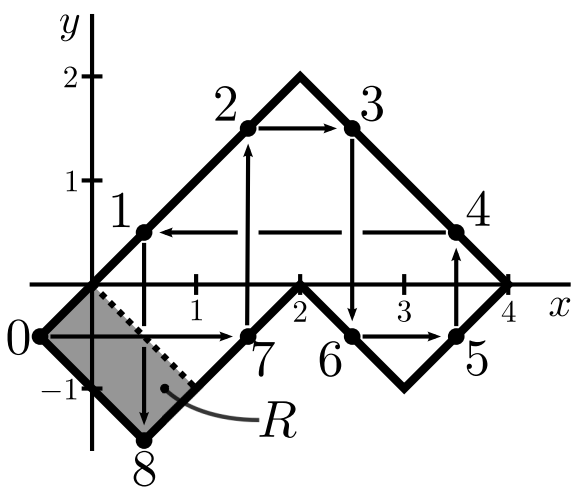

Figure 5.2. (a) Closed billiard corresponding to the closed cleaved rainbow meander of figure 5.1(c). (b) Open billiard corresponding to the open meander of figure 5.1(b). Note the shaded new rectangle $R$ attached to the lower left. Only the new corners 0 and $8=2 N$ of the new polygon are placed at half-integer coordinates $x, y$ and can therefore serve as the starting and end point of the nonclosed billiard path which represents the unbounded meander component.

5.2. Closed meanders on the 2-sphere. Consider $k$ disjoint closed $C^{1}$ Jordan curves $J_{1}, \ldots, J_{k}$ on the standard 2-sphere $S^{2}$, and another closed $C^{1}$ Jordan curve $J_{0}$ which intersects $J_{1}, \ldots, J_{k}$ transversely. Without loss of generality we may assume $J_{0}$ to be the $\pm 90^{\circ}$ meridian through the poles, after a diffeomorphism of $S^{2}$. Moreover we may assume the north pole to not coincide with any of the intersection points $1, \ldots, 2 N$. Upon standard stereographic projection to the Euclidean plane, $J_{0}$ becomes the $x$-axis and $M=J_{1} \cup \cdots \cup J_{k}$ becomes a closed plane (multi-)meander with $k$ connected components. Conversely any closed plane meander $M$ can be viewed in the one-point compactification $S^{2}$ of $\mathbb{R}^{2}$, in this way. Simple meanders, $k=1$, arise from the mutual intersections of two transverse Jordan curves $J_{0}$ and $J_{1}$.

Around 1840 Gauss considered transverse self-intersections of a single closed curve $K$ in $S^{2}$; see [Ga1840]. Label the self-intersections of $K$ by "letters" $1, \ldots, N^{\prime}$. The Gauss word problem asks for the possible words, with each letter appearing exactly twice, such that the letters appear in order as $K$ is traversed once. See [Ros99] for a contemporary account. 
For example consider the two transverse Jordan curves $J_{0}$ and $J_{1}$ above, but assume they do intersect at the north pole. In the stereographic plane this corresponds to an open meander $J_{1}$ which crosses the horizontal axis $J_{0}$ at $2 N-1$ remaining transverse intersections. Joining loose ends of $J_{0}$, $J_{1}$ in one or the other way, we obtain a closed self-transverse curve $K$. The Gauss word of $K$ which determines the topology of self-intersection then lists all $N^{\prime}=2 N-1$ intersection letters, say, first in order and then in a possibly different but parity preserving permutation $\sigma$. This permutation $\sigma$ is directly related to the Fusco-Rocha permutation, or Sturm permutation, [FuRo91] which has become of central importance for a combinatorial characterization of Sturm global attractors of the PDE (5.1); see for example [FiRo96, FiRo99, FiRo00, FiRoWo11] and the references there.

The $S^{2}$ point of view suggests an equivalence relation on closed meanders $M=J_{1} \cup \cdots \cup J_{k}$ as follows. We may place the north pole of $S^{2}$ within any of the $2 N$ subintervals of the meridian $J_{0} \backslash M$ to obtain possibly different, but equivalent, closed plane meanders $M_{1}, \ldots, M_{2 N}$ after stereographic projection. Obviously this notion of equivalence preserves the number of connected components.

For example consider a circle-free rainbow meander $M_{1}$. Placing the north pole in the cleavage interval $(N, N+1)$ of the rainbow produces another circle-free rainbow meander $M_{2}$. The associated billiards $B_{1}$ and $B_{2}$ are related by plane rotation through $180^{\circ}$ around the point $\frac{1}{2}\left(N, \beta^{ \pm}(N)\right)$.

Placing the north pole at any of the intersection points $1, \ldots, 2 N$ instead, an analogous construction applies to the open meanders as arising, e.g., in the Sturm PDE problem (5.1). We have not even started to explore the consequences of this equivalence on the level of Sturm global attractors.

Returning to the opening and closing of meanders as in figures 5.1(b),(c) we now see how our constructions can be viewed on $S^{2}$, if we identify the points 0 and $2 N$ and let them pass through the north pole. Then closing open meanders corresponds to one or the other unfolding of such a pole passage.

5.3. Temperley-Lieb algebras: traces and rainbows. Our brief exposition basically follows Di Francesco et al [dFGG97, dFG05]; see also [We95]. A Temperley-Lieb algebra $T L_{n}(\tau)$ of order $n$ with parameter $\tau$ is a matrix algebra with $N$ generators $1=e_{0}, e_{1}, \ldots, e_{N-1}$ and the relations

$$
\begin{aligned}
e_{i}^{2} & =\tau e_{i} \\
e_{i} e_{j} & =e_{j} e_{i} \\
e_{i} e_{i \pm 1} e_{i} & =e_{i}
\end{aligned}
$$



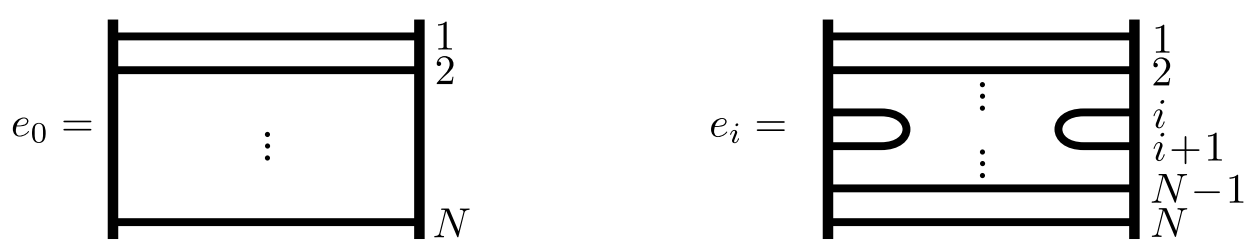

FiguRE 5.3. Diagram representations of Temperley-Lieb generators $e_{0}=1$ and $e_{i}$, for $i=1, \ldots, N-1$.

for all meaningful nonadjacent $i \neq j$; see [TeLi71]. In the $N$-strand diagram representation of Temperley-Lieb algebras $T L_{N}(\tau)$ sketched in figure 5.3, products are represented by (homotopy classes of) concatenations of $n$ strand diagrams, left to right. Resulting interior cycles are eliminated to be replaced by a prefactor of $\tau$, each, in view of property (5.4). Properties (5.5) and (5.6) are obviously satisfied by these $N$-strand diagrams.

Following [dFGG97], we define the trace of a monomial $e=e_{i_{1}} \ldots e_{i_{m}} \in$ $T L_{N}(\tau)$ as follows. In the $N$-strand diagram of $e$, we close up matching right and left ends of the same strand index. Let $k^{\prime}$ denote the resulting number of connected components and define the trace

$$
\operatorname{tr}(e):=\tau^{k^{\prime}} .
$$

Note that some of the components may be interior to $e$, whereas $1 \leq k \leq k^{\prime}$ other components involve the exterior closing. See figure 5.4(a),(b) for an illustration with $N=4, e:=e_{2} e_{1} e_{3} \in T L_{4}(\tau)$ and $\operatorname{tr}(e)=k=k^{\prime}=1$.

Figure 5.4(c) shows how the exterior connectivity $k$ of $e=e_{2} e_{1} e_{3} \in$ $T L_{4}(\tau)$ coincides with the connectivity of an equivalent rainbow meander. Indeed the interior strands of $e$ define the upper arches, and the exterior strands define the lower rainbow, when we appropriately join the vertical left and right boundaries of the strand diagram of $e$ to become the horizontal $x$-axis of the meander.

Conversely, we may pass from any $2 N$ rainbow meander $(c)$ to the $N$ strand diagram of a ( $\tau$-reduced) monomial $e \in T L_{N}(\tau)$ with $k^{\prime}=k$ exterior components. This relates the number of connected components of rainbow meanders, alias plane Cartesian billiards, to traces in Temperley-Lieb algebras.

5.4. Meanders and rainbows. Closed rainbow meanders $M_{*}$ are characterized by the nested arrangement of all $N$ lower arches $(i, 2 N+1-i)$. We have seen how circle-free rainbow meanders $M_{*}$ are equivalent to plane Cartesian billiards. The previous subsection, on the other hand, has recalled how rainbow meanders $M_{*}$ relate to traces in Temperley-Lieb algebras $T L_{N}(\tau)$. More precisely it is the number $k$ of connected components 
(a) $e:=e_{2} e_{1} e_{3} \in T L_{4}(\tau)$

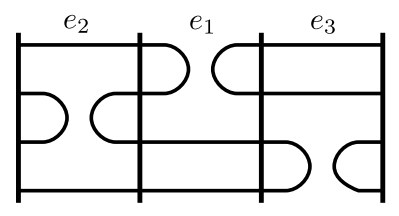

(b) $\operatorname{tr}(e)=\tau$

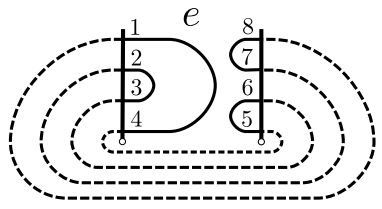

(c) rainbow meander of $e$

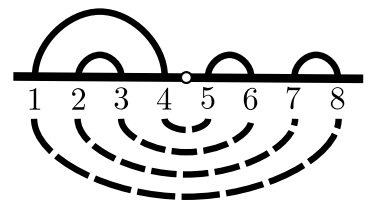

FIGURE 5.4. (a) Diagram representation of the 4-strand diagram $e=e_{2} e_{1} e_{3} \in T L_{N}(\tau), N=4$. (b) Homotopy equivalent diagram of $e$ with dashed exterior strands matching right and left ends of the same strand index. Note the number $k=1$ of exterior connected components of the closed diagram. (c) Joining the lower ends " $O$ " of the vertical strand boundaries of e, and opening the boundaries to become horizontal we obtain an equivalent rainbow meander with $2 \mathrm{~N}$ intersections and the same number $k=1$ of exterior connected components as in (b).

of the rainbow meander $M_{*}$ which determines the trace and decides, in case $M_{*}$ is simple alias $k=1$ alias $\operatorname{tr}=\tau$, whether the Cartesian billiard is transitive.

Closing the simple open Sturm meanders $\tilde{M}$ of the PDE setting (5.1), however, we mostly do not arrive at a closed rainbow meander $M_{*}$. Therefore we discuss two constructions, in this subsection, which convert general closed meanders $M$ to closed rainbow meanders $M_{*}$ without changing the connectivity $k$.

The first construction converts any closed meander $M$ to a cleaved closed rainbow $M_{*}$; in particular $M_{*}$ is circle-free. However, the number of $2 N$ intersections of the original meander $M$ with the horizontal $x$-axis is doubled to $4 N$, for the cleaved rainbow $M_{*}$. See figure 5.5 for an example with $N=4$. In general the $N$ upper arches $(i, j)$ of $M$ are kept unchanged in $M_{*}$, for $1 \leq i<j \leq 2 N$, and are located to the left of the $(2 N, 2 N+1)$ cleavage of $M_{*}$. The $N$ lower arches $(i, j)$ of $M$, however, are converted to upper arches $(4 N+1-i, 4 N+1-j)$ to the right of the cleavage of $M_{*}$. Contracting the nested lower rainbow arches of $M_{*}$ restores $M$. Therefore $M$ and $M_{*}$ possess the same number $k$ of connected components.

The second construction attempts to reduce the number $N_{*}$ of arches in the rainbow meander $M_{*}$ to become lower than the bound $N_{*}=2 N$ attained in the first construction. We assume the original meander $M$ is circle-free. Let $N_{*}$ be maximal such that $\left(N_{*}, N_{*}+1\right)$ is a lower arch of $M$. (We may consider an upper arch, just as well, if we reflect $M$ through 
(a) original meander $M$

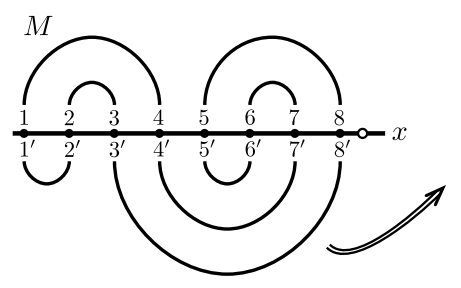

(b) cleaved rainbow meander $M_{*}$

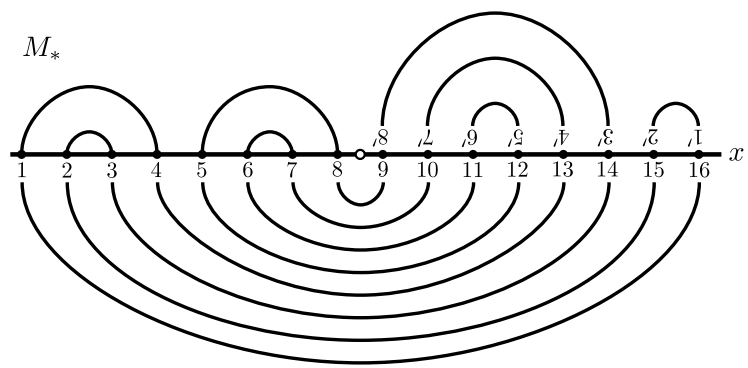

FiguRE 5.5. (a) An original closed meander $M$ with $2 N=$ 8 intersections. (b) The associated cleaved rainbow meander $M_{*}$ with $4 N=16$ intersections. Note how upper arches $(i, j)$ of $M$ have been preserved. Lower arches $(i, j)$ of $M$ have been converted to upper arches $(4 N+1-i, 4 N+1-j)$.

(a) original meander $M$

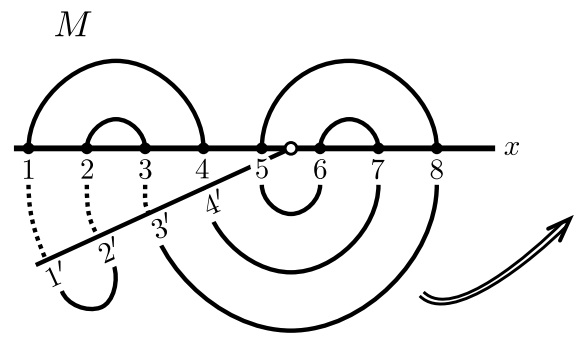

(b) shortened rainbow meander $M_{*}$

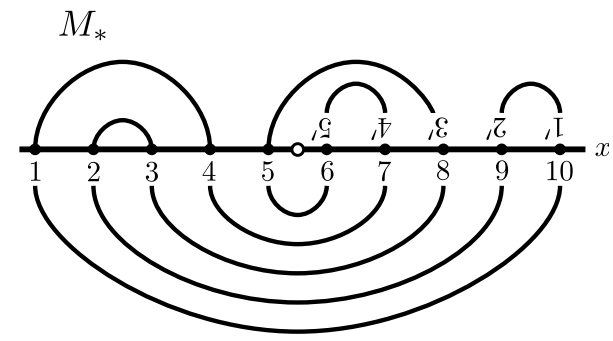

FiguRE 5.6. (a) The original closed circle-free meander $M$ with $N=4$ of figure 5.5(a), slightly opened at intersections $1, \ldots, N_{*}=5<2 N$. Note the lower rightmost innermost arch at $\left(N_{*}, N_{*}+1\right)$. (b) The fully opened rainbow meander $M_{*}$ with $N_{*}<2 N$ upper arches and $2 N_{*}<4 N$ intersections.

the $x$-axis.) As indicated in figure 5.6(a) we now replace the intersections $1, \ldots, N_{*}<2 N$ by a wedge, say with vertex at the nonintersection point $N_{*}+\frac{1}{2}$ on the $x$-axis. To arrive at the rainbow meander $M_{*}$ we open the wedge to a full $180^{\circ}$, generating the $N_{*}<2 N$ nested lower arches of the rainbow $M_{*}$. We preserve the upper and lower arches $(i, j)$ of the original meander which reside in $1, \ldots, N_{*}$ entirely. The upper and lower arches of $M$ which share an intersection point in $N_{*}+1, \ldots, 2 N$, however, are each merged into a single upper arch of $M_{*}$. 
More precisely we map an upper $\operatorname{arch}(i, j)$ of $M$ with $1 \leq i<j \leq N_{*}$ to the same upper arch of $M_{*}$. A lower arch $(i, j)$ of $M$ in the same range maps to the upper arch $\left(2 N_{*}+1-i, 2 N_{*}+1-j\right)$ of $M_{*}$. Any remaining upper $\operatorname{arch}\left(i, j_{1}\right)$ of $M$ has $i \leq N_{*}<j_{1} \leq 2 N$. It can be merged with its lower counterpart $\left(j, j_{1}\right)$ of $M$ which satisfies $j \leq N_{*}<j_{1} \leq 2 N$ by construction. Here we have used maximality of $N_{*}<2 N$. The merger provides an upper arch

$$
\left(i, 2 N_{*}+1-j\right)
$$

of $M_{*}$.

This second construction provides a circle-free rainbow meander $M_{*}$, with $N<N_{*}<2 N$ arches and $2 N_{*}<4 N$ intersections, from a circlefree rainbow meander $M$ with $N$ arches and $2 N$ intersections. In fact our construction only used that the $\operatorname{arch}\left(i, j_{1}\right)$ of $M$ is not part of a circle. Contracting the lower wedge again and re-inserting the arches of $M_{*}$ across its cleavage at $\left(N_{*}, N_{*}+1\right)$ below, we again conclude that the original circlefree meander $M$ and the shortened rainbow $M_{*}$ possess the same number $k$ of connected components.

\section{Examples: simplicity of some rainbow meanders}

For positive integers $p_{1}, \ldots, p_{n}$ we define the closed rainbow meander $M=M\left(p_{1}, \ldots, p_{n}\right)$ as follows. For any positive integer $p$ we call a configuration of $p$ nested arches a rainbow of size $p$, or a $p$-rainbow. Then the upper arches of the meander $M=M\left(p_{1}, \ldots, p_{n}\right)$ consist, left to right, of adjacent rainbows of sizes $p_{1}, \ldots, p_{n}$. The lower arches of the rainbow meander $M$ form a single rainbow, by definition, of size $N=p_{1}+\cdots+p_{n}$. Below we discuss connectivity of such rainbow meanders $M$ for $n=1,2,3$. Already the case $n=4$ is open!

The case $M=M\left(p_{1}\right)$ of $n=1$ and $p_{1}=N$ is trivial: the meander $M$ consists of $p_{1}$ nested circles.

The case $M=M(p, p)$ of $n=2$ and $p_{1}=p_{2}=p$ is a cleaved meander; see figure 6.1(a). The associated Cartesian billiard $B=B(p, p)$ is a $p \times p$ square. Again, we call the square $p \times p$ although the (Euclidean) length of its sides is $\sqrt{2} p$. The corners are at $(0,0),( \pm p, p)$, and $(2 p, 0)$; see figure 6.1(b). Obviously the cleaved rainbow meander $M(p, p)$ has $p$ connected components. The $p$ kidney shaped components are given by the rectangular billiard paths starting with the $p$ horizontal flight levels $y=i^{\prime}=i-\frac{1}{2}$ in the $p \times p$ square billiard $B$, for $i=1, \ldots, p$, respectively.

The general case $M=M\left(p_{1}, p_{2}\right)$ of $n=2$ is slightly more interesting; see figure $6.2(\mathrm{a}),(\mathrm{b})$. Without loss of generality assume $p_{1}>p_{2}$; or else interchange $p_{1}$ and $p_{2}$ by flipping the billiard $B$ through the horizontal $x$-axis. For $p_{1}>p_{2}$ the associated Cartesian billiard $B=B\left(p_{1}, p_{2}\right)$ is a rectangle 
(a) meander $M=M(p, p)$

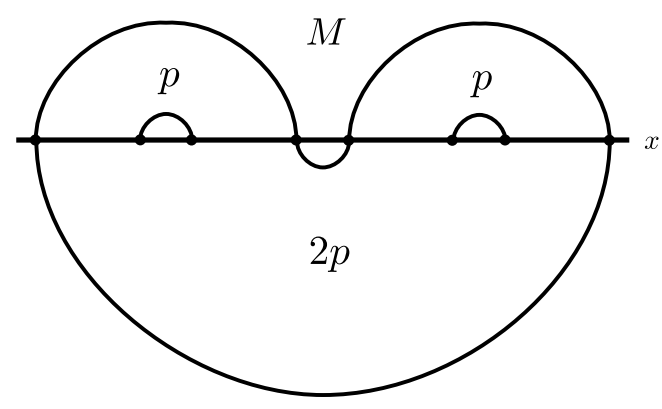

(b) equivalent square billiard $B=B(p, p)$

Figure 6.1. (a) Symmetrically cleaved rainbow meander $M=M(p, p)$. (b) Corresponding square billiard $B=$ $B(p, p)$ with $p$ closed rectangular billiard paths.

(a) meander $M=M\left(p_{1}, p_{2}\right), p_{1}>p_{2}$

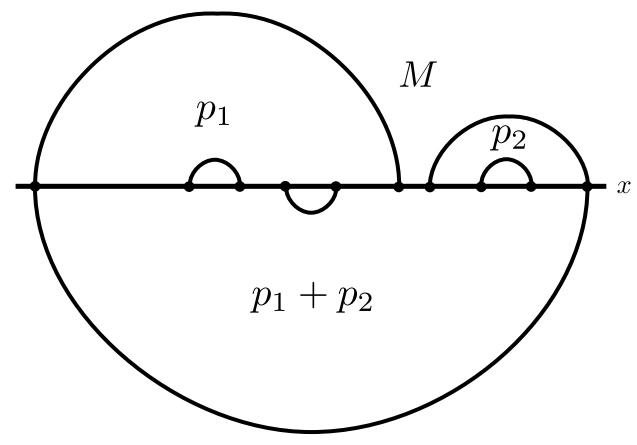

(b) equivalent rectangular billiard $B=B\left(p_{1}, p_{2}\right)$

FiguRE 6.2. Circle-free rainbow meander $M=M\left(p_{1}, p_{2}\right)$ with $p_{1}>p_{2}$, in (a), and corresponding rectangular billiard $B=B\left(p_{1}, p_{2}\right)$, in (b). Note the shaded $p_{2} \times p_{2}$ square $Q$ in the $p_{1} \times p_{2}$ rectangle $B$ with the dashed line as boundary.

with corners $(0,0),\left(p_{1}, p_{1}\right),\left(p_{2},-p_{2}\right)$, and $\left(p_{1}+p_{2}, p_{1}-p_{2}\right)$; see figure 6.2. We claim that the number $k=k\left(p_{1}, p_{2}\right)$ of connected components of the circle-free meander $M=M\left(p_{1}, p_{2}\right)$ or, equivalently, the number of paths in the Cartesian billiard $B=B\left(p_{1}, p_{2}\right)$ at half-integer levels is given by

$$
k=k\left(p_{1}, p_{2}\right)=\operatorname{gcd}\left(p_{1}, p_{2}\right),
$$

where gcd denotes the greatest common divisor. 
Proving (6.1) is particularly straightforward in the billiard setting of figure 6.2(b). Consider the shaded $p_{2} \times p_{2}$ square $Q$ to the lower left of the dashed line from $\left(2 p_{2}, 0\right)$ to $\left(p_{2}, p_{2}\right)$ in the billiard rectangle $B$. As we have already seen in our discussion of the square billiard $B(p, p)$, billiard paths inside the square $Q$ are $p_{2}$ rectangles. Cutting off the $p_{2} \times p_{2}$ square $Q$ from the $p_{1} \times p_{2}$ rectangle in fact leaves a $\left(p_{1}-p_{2}\right) \times p_{2}$ rectangle behind with the exact same number and connectivity of paths as before. Instead of entering and leaving the shaded $p_{2} \times p_{2}$ square $Q$, we simply reflect paths at the new dashed line boundary. Cutting off squares from the resulting rectangles repeatedly, in this manner, leads to a $q \times q$ square. By the Euclidean Algorithm of division with remainder, the square has side length $q=\operatorname{gcd}\left(p_{1}, p_{2}\right)$. By our study of $B(q, q)$, the original billiard $B\left(p_{1}, p_{2}\right)$ and the original rainbow meander $M\left(p_{1}, p_{2}\right)$ must therefore have $k=k\left(p_{1}, p_{2}\right)=$ $q$ connected components, i.e. as many as the reduced square $B(q, q)$. This proves claim (6.1).

(a) meander $M=M\left(p_{1}, p_{2}, p_{3}\right)$ for $p_{1}<p_{3}$

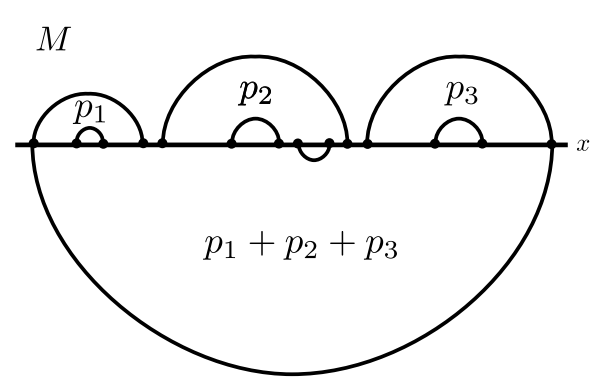

(b) equivalent L-shaped billiard $B=B\left(p_{1}, p_{2}, p_{3}\right)$

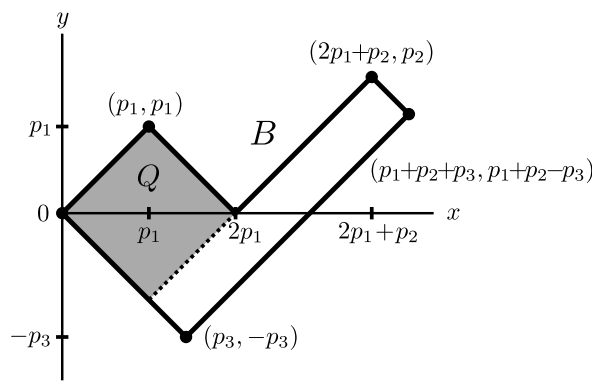

Figure 6.3. Circle-free rainbow meander $M=$ $M\left(p_{1}, p_{2}, p_{3}\right)$ with $p_{1}<p_{3}$, in (a), and corresponding $L$-shaped billiard $B=B\left(p_{1}, p_{2}, p_{3}\right)$, in (b). Note the shaded $p_{1} \times p_{1}$ square $Q$ in the left wing of $B$ with the dashed line as boundary.

As a third example we consider the case $M=M\left(p_{1}, p_{2}, p_{3}\right)$ of $n=3$ upper rainbows. The symmetric case $p_{1}=p_{3}$ with $p_{1}+p_{2}$ connected components, $p_{2}$ of them circles, is not interesting. Without loss of generality we may therefore assume $p_{1}<p_{3}$. In particular, the meander $M$ is circle-free. The associated billiard $B=B\left(p_{1}, p_{2}, p_{3}\right)$ is $L$-shaped with the six corners 0 , $\left(p_{1}, p_{1}\right),\left(p_{3},-p_{3}\right),\left(2 p_{1}, 0\right),\left(2 p_{1}+p_{2}, p_{2}\right)$, and $\left(p_{1}+p_{2}+p_{3}, p_{1}+p_{2}-p_{3}\right)$; see figure 6.3. We claim that the number $k=k\left(p_{1}, p_{2}, p_{3}\right)$ of connected components of the circle-free rainbow meander $M=M\left(p_{1}, p_{2}, p_{3}\right)$ or, equivalently, of the Cartesian billiard $B=B\left(p_{1}, p_{2}, p_{3}\right)$ is given by

$$
k=k\left(p_{1}, p_{2}, p_{3}\right)=\operatorname{gcd}\left(p_{2}+p_{1}, p_{2}+p_{3}\right) .
$$


As with (6.1), we prove (6.2) in the billiard setting of figure 6.3(b). As in our proof of (6.1) we may cut the shaded $p_{1} \times p_{1}$ square $Q$ off the left wing along the dashed line from $\left(p_{1},-p_{1}\right)$ to $\left(2 p_{1}, 0\right)$, without changing the connectivity of the Cartesian billiard. The resulting reduced rectangle has sides $p_{3}-p_{1}$ and $p_{1}+p_{2}$. Inserting (6.1) this implies

$$
\begin{aligned}
k\left(p_{1}, p_{2}, p_{3}\right) & =k\left(p_{3}-p_{1}, p_{1}+p_{2}\right)=\operatorname{gcd}\left(p_{3}-p_{1}, p_{1}+p_{2}\right)= \\
& =\operatorname{gcd}\left(p_{2}+p_{1}, p_{2}+p_{3}\right),
\end{aligned}
$$

and claim (6.2) is proved.

Reflecting the $L$-shaped billiard $B\left(p_{1}, p_{2}, p_{3}\right)$ through the wing boundaries from 0 to $\left(p_{1}, p_{1}\right)$ and from $\left(2 p_{1}+p_{2}, p_{2}\right)$ to $\left(p_{1}+p_{2}+p_{3}, p_{1}+p_{2}-p_{3}\right)$ repeatedly, by the way, we obtain a Cartesian version of a Sinai billiard: a rectangular domain with a rectangular hole, on the integer lattice, and with flight paths at $\pm 45^{\circ}$ angles to the boundary.

Of course it is tempting to extend the above elementary observations and address connectivities $k=k\left(p_{1}, \ldots, p_{n}\right)$ involving more than $n=3$ upper rainbows - not to speak of the complications of less simple-minded nesting of arches or parentheses. Looking into the case $n=4$, quite a few geometric reductions actually come to mind and many particular subcases are easily settled. The complexities of the general case seem to be such, however, that we are not able, as yet, to provide a simple formula analogous to (6.1), (6.2), even for the case $n=4$. And of course there are many further cases to explore.

In summary we find it intriguing how many puzzles are still in store, even in a supposedly elementary subject like the combinatorics of Jordan curves and billiards in the plane.

\section{References}

[Ar88] V.I. Arnold. A branched covering $C P^{2} \rightarrow S^{4}$, hyperbolicity and projective topology. Sib. Math. J. 29 (1988) 717-726.

[ArVi89] V.I. Arnold and M.I. Vishik et al. Some solved and unsolved problems in the theory of differential equations and mathematical physics. Russian Math. Surveys 44 (1989) 157-171.

[BrCh84] P. Brunovský and S.-N. Chow. Generic properties of stationary state solutions of reaction-diffusion equations. J. Differential Eqs. 53 (1984) $1-23$.

[dFGG97] E. Di Francesco, O. Golinelli and E. Guitter. Meanders and the Temperley-Lieb algebra. Commun. Math. Phys. 186 (1997) 1-59.

[dFG05] P. Di Francesco and E. Guitter. Geometrically constrained statistical systems on regular and random lattices: From folding to meanders. Physics Reports 415 (2005) 1-88.

[Fi94] B. Fiedler. Global attractors of one-dimensional parabolic equations: sixteen examples. Tatra. Mt. Math. Publ. 4 (1994) 67-92. 
[FiRo96] B. Fiedler and C. Rocha. Heteroclinic orbits of semilinear parabolic equations. J. Differential Eqs. 125 (1996) 239-281.

[FiRo99] B. Fiedler and C. Rocha. Realization of meander permutations by boundary value problems. J. Differential Eqs. 156 (1999) 282-308.

[FiRo00] B. Fiedler and C. Rocha. Orbit equivalence of global attractors of semilinear parabolic differential equations. Trans. Amer. Math. Soc. 352 (2000) 257-284.

[FiRo09] B. Fiedler and C. Rocha. Connectivity and design of planar global attractors of Sturm type. I: Bipolar orientations and Hamiltonian paths. J. Reine Angew. Math. 635 (2009) 71-96.

[FiRo13] B. Fiedler and C. Rocha. Schoenflies spheres as boundaries of bounded unstable manifolds in gradient Sturm systems. J. Dyn. Differential Eqs. (2013), to appear.

[FiSc02] B. Fiedler and A. Scheel. Dynamics of reaction-diffusion patterns, in: M. Kirkilionis, R. Rannacher and F. Tomi (Eds.). Trends in Nonlinear Analysis. Festschrift dedicated to Willi Jäger for his 60th birthday. Springer-Verlag, Heidelberg, 2002, 23-152.

[FiRoWo11] B. Fiedler, C. Rocha and M. Wolfrum. A permutation characterization of Sturm global attractors of Hamilton type. J. Differential Eqs. 252 (2011) 588-623.

[FuRo91] G. Fusco and C. Rocha. A permutation related to the dynamics of a scalar parabolic PDE. J. Differential Eqs. 91 (1991) 75-94.

[Ga1840] C.F. Gauss. Gesammelte Werke VIII, Nachlass, I. Zur Geometria Situs. (ca. 1840) 271-286.

[Gi98] M. Gimbutas. Die Sprache der Göttin. Zweitausendeins, Frankfurt/M., 1998.

[KB70] E. Klengel-Brandt. Reise in das alte Babylon. Prisma-Verlag Zenner und Gürchott, Leipzig, 1970.

[Ro91] C. Rocha. Properties of the attractor of a scalar parabolic PDE. J. Dyn. Differential Eqs. 3 (1991) 575-591.

[Ro94] C. Rocha. Bifurcation in discretized reaction-diffusion equations. Resenhas IME-USP 1 (1994) 403-419.

[Ro07] C. Rocha. Realization of period maps of planar Hamiltonian systems. J. Dyn. Differential Eqs. 19 (2007) 571-591.

[Ros99] P. Rosenstiehl. A new proof of the Gauss interlace conjecture. Advances in Applied Mathematics 23 (1999) 3-13.

[Sa03] J. Saward. Labyrinths 83 Mazes. Gaia Books, London, 2003, p. 97.

[SmTrWa80] J. Smoller, A. Tromba and A. Wassermann. Non-degenerate solutions of boundary-value problems. Nonl. Analysis 4 (1980) 207-216.

[TeLi71] H.N.V. Temperley and E.H. Lieb. Relations between the "percolation" and "colouring" problem and other graph theoretical problems associated with regular plane lattices: Some exact results for the percolation problem. Proc. Roy. Soc. London Ser. A 322 (1971) 251-280.

[We95] B.W. Westbury. The representation theory of the Temperley-Lieb algebras. Math. Z. 219 (1995) 539-565. 